\title{
Recurrent Ovarian Cyst in Pregnancy, The Advantage of Laparoscopic Approach - A Case Report
}

\author{
Wan Ahmad Hazim Wan Ghazali*, Emily Christine D Silva and Ser Yee Ning \\ Department of Obstetrics and Gynecology, Putrajaya Hospital, Malaysia
}

Received: 阱 August 05, 2018; Published: 制 August 13, 2018

*Corresponding author: Wan Ahmad Hazim Wan Ghazali, Putrajaya Hospital, Pusat Pentadbiran Kerajaan Persekutuan, Presint 7 , 62250 Putrajaya, Malaysia

\begin{abstract}
Background: The incidence of recurrent ovarian cyst in pregnancy is rare. However, complications such as torsion, rupture, infection, haemorrhage and obstructed labour may occur. Thus, surgical removal is required to avoid such complications.

Case: A 27-year-old parity 1 with history of laparoscopic cystectomy during her first pregnancy presented with recurrent ovarian cyst in her second pregnancy at 13 weeks of gestation. Removal of ovarian cyst was done laparoscopically without significant complications. The remaining of her pregnancy was uncomplicated, and she delivered a healthy infant at term.
\end{abstract}

Conclusion: Laparoscopic cystectomy is safe and should be the gold standard in pregnant women.

Keywords: Laparoscopy; Minimal invasive surgery; Recurrent ovarian cyst; Pregnancy

\section{Background}

The incidence of recurrent ovarian cyst is rare and may occur on the same or contralateral ovary which may require repeated surgery. Ovarian cyst with diameter $\geq 5 \mathrm{~cm}$ is at risk of cyst accidents such as torsion, rupture, infection and haemorrhage. [1] These complications may necessitate emergency surgery which carries a higher risk of foetal loss and maternal morbidity as compare with elective surgery. Furthermore, 17-21\% of cases have obstructed labour due to the presence of cyst. [2] Laparoscopic cystectomy in pregnancy is a surgical alternative to conventional laparotomy. Its main advantage lies within its power of magnification and panoramic view of the pelvis allowing lesser intra-operative uterine manipulation. This further translates to shorter hospital stay with faster recovery, better cosmetic outcome, reduced postoperative pain and complications such as bleeding and pelvic adhesion. [2-4] There are also evidence suggesting that laparoscopy and laparotomy do not differ in foetal outcome (foetal weight, growth restriction, foetal malformations and infant survival rates). [3] Here we present a case of recurrent ovarian cyst in pregnancy which was managed laparoscopically.

\section{Case Summary}

A 27 years old primigravida was referred for an asymptomatic uniloculated clear cyst diagnosed for 6 weeks gestation measuring
$5 \mathrm{~cm}$ which persisted. She was counselled and consented to laparoscopic cystectomy under general anaesthesia at 13 weeks gestation. Intra-operatively, the Veress needle was inserted through the umbilicus away from the fundus followed by primary $5 \mathrm{~mm}$ port after Pneumoperitoneum created with $\mathrm{CO} 2$. Two other $5 \mathrm{~mm}$ ports were placed at left lumbar and Palmer's point and intra-abdominal pressure was reduced to $12-15 \mathrm{mmHg}$. A gravid uterus was seen with normal left fallopian tube and ovary. The peritoneal cavity, bowel and liver surface were normal with no adhesions, endometriotic spots or ascitic fluid. A benign-looking right ovarian cyst with smooth surface was seen measuring $5 \times 6 \mathrm{~cm}$. An incision was made across the thicker surface of the cyst with monopolar spade and the cyst dissected away from ovarian tissue using non-traumatic graspers. The cyst was then placed into an Endo-bag and removed via a $10 \mathrm{~mm}$ port which was converted from a $5 \mathrm{~mm}$ port at the left iliac fossa without spillage of cyst content into the pelvic cavity. The remaining ovarian tissue was refashioned using non-absorbable Prolene 3/0 and hemostasis secured. Peritoneal cavity washed with $1000 \mathrm{cc}$ normal saline. The whole procedure was completed within 35 minutes with very minimal blood loss. Prophylactic antibiotic was administered as a single dose prior to surgery. No tocolytic agents were given post-operatively. Trans-abdominal ultrasound done before and after surgery showed viable foetus. 
Her postoperative recovery was uncomplicated, and she was discharged home the following day. Histopathology report confirmed a benign serous cyst. Her remaining antenatal course was uneventful, and she delivered a healthy baby boy vaginally, weighing $3.13 \mathrm{~kg}$ with good Apgar score. A year later, she was referred at 10 weeks of gestation in her second pregnancy with recurrent ovarian cyst measuring $5 \mathrm{~cm}$ at the left adnexa. She was otherwise asymptomatic. She consented for a second laparoscopic cystectomy in view of increased cyst size to $7 \mathrm{~cm}$. Laparoscopic left cystectomy was done at 13 weeks of gestation under general anaesthesia. All ports were placed through the previous port site scars. Intra-operative findings were relative unremarkable except for a benign-looking left ovarian cyst with smooth surface measuring $6 \times 6 \mathrm{~cm}$ was noted. There no adhesions noted. Similar technique was employed as before and the intact cyst was removed without spillage. The whole procedure was completed within 30 minutes with minimal blood loss. She was discharged well without tocolytic agents the following day with viable foetus on transabdominal scan. Histopathology report confirmed an inclusion cyst. She delivered a healthy baby girl vaginally, weighing $3.26 \mathrm{~kg}$ with good Apgar score.

\section{Discussion}

Recurrent ovarian cyst in pregnancy requiring repeated surgical removal is rare. Laparoscopic approach allows pregnant mothers to avoid repeated laparotomy within a short period which carries a higher risk of miscarriage and maternal morbidities. In worst case scenarios, mothers may end up with 4 major laparotomies within a short period of time, if both her pregnancies were delivered by caesarean sections and both her ovarian cyst were dealt with via laparotomy. The use of laparoscopic approach can reduce the event of major surgery to at least $50 \%$, if the mother had delivered both deliveries were by caesarean section. In this case, the mental trauma, anguish and anxiety were avoided. The patient was receptive to treatment and willing to be subjected to a second cystectomy because her previous surgery was not traumatising.

Laparoscopic has proven to cause lesser post-operative complications (pain, bleeding, pelvic adhesion) with faster recovery and does not affect the foetus. We found no adhesions intra-operatively during second surgery. No new scars were made during second surgery as ports were placed through previous scar site. Pain and discomfort of stretching and distension of scar due to rapidly growing uterus was non-existence. This is inline with pain-free surgery, a concept defined in our hospital and ministry of health which consist of pain control by acupuncture, minimal invasive surgery and liberal use of acute pain service. Laparoscopic surgery should be the gold standard in approaching all ovarian cysts in pregnancy as it has shown to be more superior and beneficial to this group of women. Laparotomies in pregnancy must be avoided as much as possible due to the potential mental and physical morbidities as mention before. We should continue to work towards the goal of achieving pain-free surgery and avoid inflicting pain and morbidities for patients' benefit.

\section{References}

1. Al-Fozan H, Tulandi $\mathrm{T}$ (2002) Safety and risks of laparoscopy in pregnancy. CurrOpinObstetGynecol 14(4): 375-379.

2. Carmen R Nezhat, SenolKalyoncu, Ceana H Nezhat, Ehrin Johnson, N Berlanda, et al. (1999) Laparoscopic Management of ovarian dermoid cysts: ten years' experience. JSLS 3(3): 179-184.

3. Mendilcioglu I, Zorlu CG, Trak B, Ciftei C, Akinci Z (2002) Laparpscopic management of adnexal masses, Safety and effectiveness. J Reprod Med 47(1): 36-40.

4. Nezhat C, Winer W, Nezhat F (1989) Laparoscopic removal of dermoid cyst. Obstet Gynecol 73(2): 278-281.
This work is licensed under Creative Commons Attribution 4.0 License

Submission Link: Submit Article

DOI: $10.32474 /$ OAJRSD.2018.01.000114

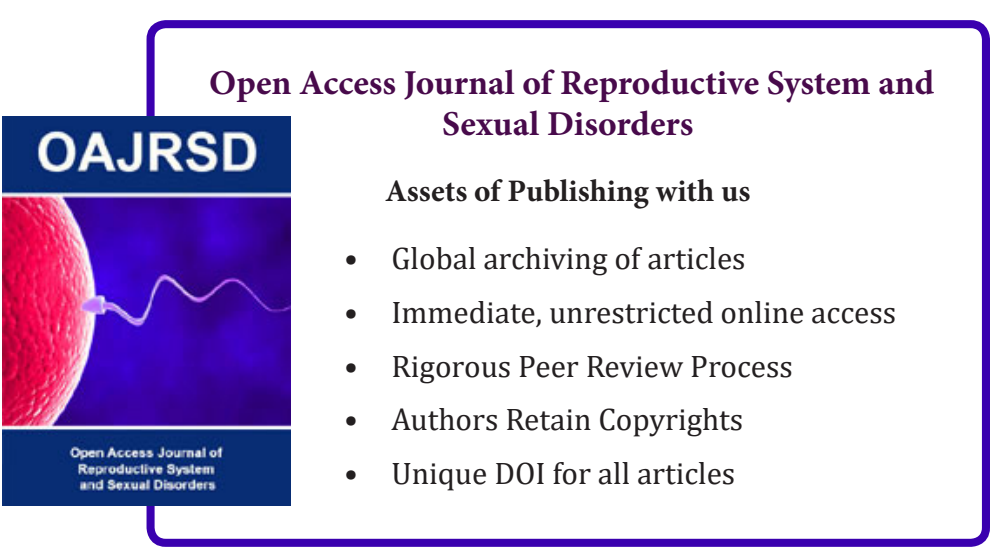

